\title{
Local symmetry on almost Kenmotsu three-manifolds
}

\author{
Jong Taek CHO
}

(Received June 9, 2014; Revised Septemer 22, 2014)

\begin{abstract}
We prove that a locally symmetric almost Kenmotsu three-manifold is locally isometric to either the hyperbolic space $\mathbb{H}^{3}(-1)$ or a product space $\mathbb{H}^{2}(-4) \times \mathbb{R}$.

Key words: almost Kenmotsu 3-manifold, local symmetry.
\end{abstract}

\section{Introduction}

Let $M$ be a manifold of odd dimension $m=2 n+1$. Then $M$ is said to be an almost contact manifold if its structure group $\mathrm{GL}_{\mathrm{m}}(\mathbb{R})$ of the linear frame bundle is reducible to $\mathrm{U}(n) \times\{1\}$. This is equivalent to the existence of an endomorphism field $\varphi$, a vector field $\xi$ and a 1-form $\eta$ satisfying

$$
\varphi^{2}=-I+\eta \otimes \xi, \quad \eta(\xi)=1
$$

We call $\xi$ the Reeb vector field. Then $M$ admits a Riemannian metric $g$ satisfying

$$
g(\varphi X, \varphi Y)=g(X, Y)-\eta(X) \eta(Y)
$$

for any vector fields $X, Y$ on $M$. Such $(M ; \varphi, \xi, \eta, g)$ is said to be an almost contact metric manifold. The fundamental 2-form $\Phi$ is defined by $\Phi(X, Y)=g(X, \varphi Y)$. If $M$ satisfies in addition $d \eta=\Phi$, then $M$ is called a contact metric manifold. An almost contact metric manifold $M$ is said to be almost Kenmotsu if $d \eta=0$ and $d \Phi=2 \eta \wedge \Phi$. The warped products of an almost Kählerian manifold and a real line give examples of almost Kenmotsu manifolds.

For an almost contact structure $(\varphi, \xi, \eta)$ of $M$, the normality defined as follows. One may define naturally an almost complex structure $J$ on $M \times \mathbb{R}$ by

2000 Mathematics Subject Classification : 53B20, 53C25, 53C35. 


$$
J\left(X, f \frac{d}{d t}\right)=\left(\varphi X-f \xi, \eta(X) \frac{d}{d t}\right)
$$

where $X$ is a vector field tangent to $M, t$ the coordinate of $\mathbb{R}$ and $f$ a function on $M \times \mathbb{R}$. If the almost complex structure $J$ is integrable, $M$ is said to be normal. A normal almost Kenmotsu manifold is said to be Kenmotsu. It is known that a normal almost contact metric structure has the CR-integrable structure (cf. [1]). For further properties and examples of (almost) Kenmotsu manifolds, we refer to [4] and [5].

E. Boeckx and the present author [2] proved that a locally symmetric contact metric manifold is either normal and of constant curvature 1 or locally isometric to the product space $\mathbb{R}^{\mathrm{n}+1} \times \mathbb{S}^{\mathrm{n}}(4)$. On the other hand, $K$. Kenmotsu [5] proved that a locally symmetric Kenmotsu manifold $M^{2 n+1}$ is locally isometric to the hyperbolic space $\mathbb{H}^{2 \mathrm{n}+1}(-1)$ (of constant curvature $-1)$. Due to G. Dileo and A. M. Pastore [4] we know that if an almost Kenmotsu manifold is locally symmetric and in addition the curvature tensor $R$ satisfies $R(X, Y) \xi=0$ for any vector fields $X, Y$ orthogonal to $\xi$, then it is either a Kenmotsu manifold of constant curvature -1 or locally the product space $\mathbb{H}^{\mathrm{n}+1}(-4) \times \mathbb{R}^{\mathrm{n}}$. Then, in the same paper [4] they raised a following question: is a locally symmetric almost Kenmotsu manifold either a Kenmotsu manifold of constant curvature -1 or locally isometric to the product $\mathbb{H}^{\mathrm{n}+1}(-4) \times \mathbb{R}^{\mathrm{n}}$ ? Very recently, Y. Wang and X. Liu [8] gave a partial affirmative answer to the question under some additional conditions. Namely, they proved that if an almost Kenmotsu manifold $M^{2 n+1}$ with $n>1$ has the CR-integrable structure and it is locally symmetric, then $M$ is locally isometric to either a Kenmotsu manifold of constant curvature -1 or locally isometric to the product $\mathbb{H}^{\mathrm{n}+1}(-4) \times \mathbb{R}^{\mathrm{n}}$. As a crucial part in their proof, they proved that for a locally symmetric almost Kenmotsu manifold with CR-integrable structure the Reeb vector field $\xi$ is an eigenvector of the Ricci operator, when $n>1$ (Lemma 4.3 in [8]). In the present paper, we prove the three-dimensional case.

Main Theorem An almost Kenmotsu three-manifold is locally symmetric if and only if it is locally isometric to the hyperbolic space $\mathbb{H}^{3}(-1)$ or a product space $\mathbb{H}^{2}(-4) \times \mathbb{R}$.

In the proof of our main theorem, we find that for locally symmetric almost Kenmotsu three-manifolds the Reeb vector field $\xi$ is an eigenvector 
of the Ricci operator (Corollary 8).

\section{Almost Kenmotsu three-manifolds and local symmetry}

All manifolds in the present paper are assumed to be connected and of class $C^{\infty}$. Let $(M ; \varphi, \xi, \eta, g)$ be a 3 -dimensional almost Kenmotsu manifold. We prepare some fundamental formulas which are mainly established in [4] (we refer also to $[6]$ ). Then we have

$$
\nabla_{\xi} \xi=0, \quad \nabla_{\xi} \varphi=0
$$

and

$$
\nabla_{X} \xi=X-\eta(X) \xi-\varphi h X
$$

for any vector field $X$ on $M$ where $h=(1 / 2) £_{\xi} \varphi$. We find that the linear operator $h$ is self-adjoint and moreover we have

$$
h \varphi+\varphi h=0, \quad h \xi=0, \quad \operatorname{tr} h=0 .
$$

From (2.2) and (2.3) we obtain that $\operatorname{div} \xi=2$. Then we observe that $a$ compact manifold does not admit almost Kenmotsu structure.

Proposition 1 ([6]) An almost Kenmotsu three-manifold is normal if and only if $h=0$. In such a case, the canonical foliation $\mathcal{F}$, which is generated by the contact distribution $D=k e r \eta$, yields a totally umbilical foliation.

We note that $\mathcal{F}$ is Riemannian, but the 1-dimensional foliation $\mathcal{F}^{\perp}$ can not be Riemannian.

For a Kenmotsu three-manifold $M$, we have from (2.2)

$$
R(X, Y) \xi=\eta(X) Y-\eta(Y) X,
$$

and

$$
S \xi=-2 \xi \text {. }
$$

Remind that the curvature tensor $R$ of a 3-dimensional Riemannian manifold is expressed by 


$$
\begin{aligned}
R(X, Y) Z= & \rho(Y, Z) X-\rho(X, Z) Y+g(Y, Z) S X-g(X, Z) S Y \\
& -\frac{r}{2}\{g(Y, Z) X-g(X, Z) Y\}
\end{aligned}
$$

for all vector fields $X, Y, Z$, where $\rho(Y, X)=g(S Y, X)$ and $r$ denotes the scalar curvature. Then together with (2.4) we have

Proposition 2 For a Kenmotsu three-manifold we have the Ricci operator:

$$
S=\left(1+\frac{r}{2}\right) I-\left(3+\frac{r}{2}\right) \eta \otimes \xi
$$

where I denotes the identity transformation.

Theorem 3 ([3]) A Kenmotsu three-manifold $M$ satisfies $\nabla_{\xi} S=0$ if and only if $M$ is locally isometric to the hyperbolic space $\mathbb{H}^{3}(-1)$.

Corollary 4 ([5]) A locally symmetric Kenmotsu three-manifold is locally isometric to the hyperbolic space $\mathbb{H}^{3}(-1)$.

Now we prove

Theorem 5 A 3-dimensional almost Kenmotsu manifold $M$ is locally symmetric if and only if $M$ is locally isometric to either the hyperbolic space $\mathbb{H}^{3}(-1)$ or a product space $\mathbb{H}^{2}(-4) \times \mathbb{R}$.

Proof. Let $M=\left(M^{3} ; \varphi, \xi, \eta, g\right)$ be an almost Kenmotsu three-manifold. Suppose that $M$ is locally symmetric. Then, in case that $M$ is normal we already know that $M$ is locally isometric to the hyperbolic space $\mathbb{H}^{3}(-1)$. Now suppose that $h$ is not identically zero. We consider on $M$ the maximal open subset $U_{1}$ on which $h \neq 0$ and the maximal open subset $U_{2}$ on which $h$ is identically zero. $U_{1} \cup U_{2}$ is open and dense in $M$. Then $U_{1}$ is non-empty and there is a local orthonormal frame field $\left\{e_{1}=e, e_{2}=\varphi e, e_{3}=\xi\right\}$ on $U_{1}$ such that $h\left(e_{1}\right)=\mu e_{1}, h\left(e_{2}\right)=-\mu e_{2}$ for some positive function $\mu$. From now our arguments will be done in $U_{1}$. Then, using the 2nd equation of (2.1) and (2.2) we have 
Lemma $6([3])$

$$
\begin{aligned}
& \nabla_{\xi} \xi=0, \quad \nabla_{\xi} e_{1}=a e_{2}, \quad \nabla_{\xi} e_{2}=-a e_{1}, \\
& \nabla_{e_{1}} \xi=-\mu e_{2}+e_{1}, \quad \nabla_{e_{1}} e_{1}=-b e_{2}-\xi, \quad \nabla_{e_{1}} e_{2}=b e_{1}+\mu \xi, \\
& \nabla_{e_{2}} \xi=-\mu e_{1}+e_{2}, \quad \nabla_{e_{2}} e_{1}=c e_{2}+\mu \xi \quad \nabla_{e_{2}} e_{2}=-c e_{1}-\xi \text {, }
\end{aligned}
$$

where $a, b, c$ are smooth functions.

From (2.8) we compute the Poisson brackets:

$$
\left[e_{3}, e_{1}\right]=(a+\mu) e_{2}-e_{1}, \quad\left[e_{1}, e_{2}\right]=b e_{1}-c e_{2}, \quad\left[e_{2}, e_{3}\right]=(a-\mu) e_{1}+e_{2} \text {. }
$$

From the Jacobi identity

$$
\left[e_{3},\left[e_{1}, e_{2}\right]\right]+\left[e_{1},\left[e_{2}, e_{3}\right]\right]+\left[e_{2},\left[e_{3}, e_{1}\right]\right]=0
$$

we get the following:

$$
\left\{\begin{array}{l}
-e_{1} \mu+e_{3} b+e_{1} a+c(a-\mu)+b=0 \\
e_{2} \mu-e_{3} c+e_{2} a+b(a+\mu)-c=0
\end{array}\right.
$$

By using (2.8) and (2.9), we compute the Riemann curvature tensor $R(X, Y) Z=\left[\nabla_{X}, \nabla_{Y}\right] Z-\nabla_{[X, Y]} Z$, and then we have the Ricci curvature tensor $\rho(X, Y)$ :

$$
\left\{\begin{array}{l}
\rho\left(e_{3}, e_{3}\right)=-2\left(\mu^{2}+1\right) \\
\rho\left(e_{3}, e_{1}\right)=\rho\left(e_{1}, e_{3}\right)=-e_{2} \mu-2 b \mu \\
\rho\left(e_{3}, e_{2}\right)=\rho\left(e_{2}, e_{3}\right)=-e_{1} \mu-2 c \mu \\
\rho\left(e_{1}, e_{1}\right)=-e_{1} c-e_{2} b-b^{2}-c^{2}-2 a \mu-2 \\
\rho\left(e_{1}, e_{2}\right)=\rho\left(e_{2}, e_{1}\right)=\xi \mu+2 \mu \\
\rho\left(e_{2}, e_{2}\right)=-e_{1} c-e_{2} b-b^{2}-c^{2}+2 a \mu-2
\end{array}\right.
$$

where we have used (2.10).

Since $M$ satisfies $\nabla R=0$, we first find that $\nabla S=0$ and the scalar curvature $r$ is constant. Since $\rho_{33}=-2\left(\mu^{2}+1\right)$, from (2.8) we obtain $e_{3} \mu=0$. Then we get 


$$
\rho_{12}=2 \mu
$$

where we have put $\rho_{i j}=\rho\left(e_{i}, e_{j}\right)$ for $i, j=1,2,3$. Differentiating (2.12) covariantly along $\xi$ again, then since $\nabla_{3} \rho_{12}=0$ we get

$$
a\left(\rho_{11}-\rho_{22}\right)=0
$$

where $a=g\left(\nabla_{e_{3}} e_{1}, e_{2}\right)$. Together (2.11) and (2.13) we find that $a=0$ and moreover we have

$$
\rho_{11}=\rho_{22}=1+\mu^{2}+\frac{r}{2}
$$

Then we prove

Lemma $7 \mu$ is constant.

Proof. Differentiating $\rho_{i i}$ covariantly for $e_{i}, i=1,2$, respectively, then since $\nabla_{i} \rho_{j k}=0$ using $(2.8)$ and $(2.11)$ we get

$$
e_{2} \mu=\mu\left(e_{1} \mu\right), \quad e_{1} \mu=\mu\left(e_{2} \mu\right)
$$

respectively. From the two equations in (2.15) we have $e_{1} \mu=e_{2} \mu=0$. Thus, together with $\xi \mu=0$ we have $\mu$ is constant along $M$.

Differentiating (2.12) covariantly for $e_{1}, e_{2}$, respectively, then using (2.8), (2.11) and Lemma 7 we have

$$
\mu \rho_{13}=\rho_{23}, \quad \mu \rho_{23}=\rho_{13},
$$

respectively. From (2.16) we have either $\mu=1$ (assuming $\mu>0$ ) or $\xi$ is an eigenvector of the Ricci operator $S$. Now we divide our arguments into two cases.

First, we consider the case $e_{3}=\xi$ is an eigenvector of the Ricci operator $S$, that is, $S e_{3}=\sigma e_{3}$, where $\sigma=-2 \mu^{2}-2$. Then from (2.11) we see that $b=c=0$ and $\rho_{11}=\rho_{22}=-2$. Moreover, the bracket relations (2.9) is reduced to

$$
\left[e_{3}, e_{1}\right]=\mu e_{2}-e_{1}, \quad\left[e_{1}, e_{2}\right]=0, \quad\left[e_{2}, e_{3}\right]=-\mu e_{1}+e_{2} .
$$

Then, owing to J. Milnor's result ([7]) we find that $M$ is locally isometric to 
a non-unimodular Lie group. That is, its Lie algebra $\mathfrak{m}$ is not unimodular and its unimodular kernel $\mathfrak{a}=\left\{X \in \mathfrak{m}\right.$ : trace $\left.a d_{X}=0\right\}$ is 2-dimensional and abelian. Indeed, $\left\{e_{1}, e_{2}\right\}$ is a basis of $\mathfrak{a}$ and trace $a d_{e_{3}}=-2 \neq 0$. The Ricci operator $S$ is represented as:

$$
\begin{aligned}
& S e_{1}=-2 e_{1}+2 \mu e_{2}, \\
& S e_{2}=2 \mu e_{1}-2 e_{2}, \\
& S e_{3}=\left(-2 \mu^{2}-2\right) e_{3},
\end{aligned}
$$

If $M$ is irreducible, then it is Einstein. If $M$ is a locally a product space of three 1-dimensional manifolds, then it is locally flat. But, from Theorem 4 in [4] we see that $h$ vanishes for an almost Kenmotsu manifold of constant sectional curvature. So, the both cases cannot occur. Thus, $M$ should be a locally product space of $\mathbb{R}$ and 2-dimensional manifold $N^{2}$ of constant curvature $k$. Then, solving the eigenvalue and eigenvector of Ricci operator $S$, we have

$$
\begin{aligned}
& S \tilde{e}_{1}=(-2+2 \mu) \tilde{e}_{1}, \\
& S \tilde{e}_{2}=(-2-2 \mu) \tilde{e}_{2}, \\
& S \tilde{e}_{3}=\left(-2 \mu^{2}-2\right) \tilde{e}_{3},
\end{aligned}
$$

where $\tilde{e}_{1}=(1 / \sqrt{2})\left(e_{1}+e_{2}\right), \tilde{e}_{2}=(1 / \sqrt{2})\left(-e_{1}+e_{2}\right)$ and $\tilde{e}_{3}=e_{3}$. From (2.18) we see that $\tilde{e}_{1}$ is tangent to $\mathbb{R}$ and $\left\{\tilde{e}_{2}, \tilde{e}_{3}\right\}$ span $T N$. Hence $\mu=1$ and $k=-4$.

Next, we consider the case $\mu=1$. Then from (2.11) and (2.16) it follows that $b=c$ and $\rho_{13}=\rho_{23}=-2 b$. Also, from (2.10) we have $e_{3} b=0$. If we differentiate $\rho_{13}=-2 b$ covariantly for $e_{1}$ and $e_{2}$, then we have $e_{1} b=-e_{2} b$ and $\rho_{11}=-2\left(e_{1} b\right)-2 b^{2}-2$. If we differentiate $\rho_{23}=-2 b$ covariantly for $e_{1}$, then we have $\rho_{22}=2\left(e_{1} b\right)-2 b^{2}-2$. But, we already know that $\rho_{11}=\rho_{22}$. Thus we have $e_{1} b=e_{2} b=0$. After all, we have that $b=c$ are constants and $\rho_{11}=\rho_{22}=-2 b^{2}-2$. Now we compute $\nabla_{1} \rho_{11}=e_{1} \rho_{11}+2 b \rho_{12}$. Then, since $\rho_{12}=2$ we have that $b=0$. This yields that $\xi$ is an eigenvector of the Ricci operator $S$. Thus, we conclude that $M$ is locally isometric to $\mathbb{H}^{2}(-4) \times \mathbb{R}$. This completes the proof.

Corollary 8 The Reeb vector field of a locally symmetric almost Kenmotsu three-manifold is an eigenvector of the Ricci operator. 
Acknowledgement The author thanks to a referee for careful reading the manuscript and useful comments for the revised version. This research was supported by Basic Science Research Program through the National Research Foundation of Korea(NRF) funded by the Ministry of Education, Science and Technology (2014R1A1A2053665).

\section{References}

[1] Blair D. E., Riemannian geometry of contact and symplectic manifolds. Second edition, Progr. Math. 203, Birkhäuser Boston, Inc., Boston, MA, 2010.

[ 2 ] Boeckx E. and Cho J. T., Locally symmetric contact metric manifolds. Monatsh. Math. 148 (2006), 269-281.

[ 3 ] Cho J. T. and Kimura M., Reeb flow symmetry on almost contact threemanifolds. Diff. Geom. Appl. 35 (2014), 266-273.

[ 4 ] Dileo G. and Pastore A. M., Almost Kenmotsu manifolds and local symmetry. Bull. Belg. Math. Soc.-Simon Stevin 14 (2007), 343-354.

[ 5 ] Kenmotsu K., A class of contact Riemannian Manifolds. Tôhoku Math. J. 24 (1972), 93-103.

[ 6 ] Kim T. W. and Pak H. K., Canonical foliations of certain classes of almost contact metric structures. Acta Math. Sinica 21 (2005), 841-846.

[ 7 ] Milnor J., Curvature of left invariant metrics on Lie groups. Adv. in Math. 21 (1976), 293-329.

[ 8 ] Wang Y. and Liu X., Locally symmetric CR-integrable almost Kenmotsu manifolds. Mediterr. J. Math. 12 (2015), 159-171.

Department of Mathematics

Chonnam National University

Gwangju 61186, Korea

E-mail: jtcho@chonnam.ac.kr 